
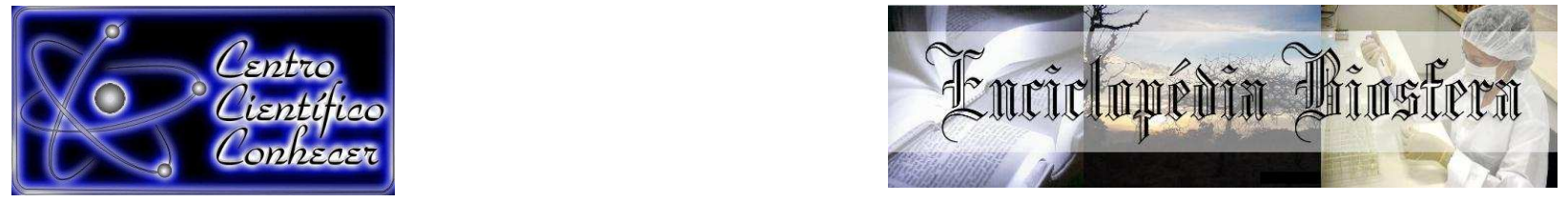

\title{
COMPARAČ̃̃ DE CURVA DE APRENDIZADO EM OVARIOSALPINGOHISTERECTOMIA POR TÉCNICA CONVENCIONAL E ABRAÇADEIRA EM AMBIENTE ACADÊMICO
}

Maria Júlia Deconto ${ }^{1}$; Marina Prazeres Moreira ${ }^{1}$; Kaue Cesar Rossi ${ }^{1}$; Amanda D'avila Verardi ${ }^{2}$; Débora Cristina Olsson ${ }^{2}$.

${ }^{1}$ Discente, Medicina Veterinária, Instituto Federal Catarinense-IFC-Concórdia, Concórdia, SC, Brasil.

${ }^{2}$ Doutor, Docente, Instituto Federal Catarinense-IFC-Concórdia, Concórdia, SC, Brasil (debora.olsson@ifc-concordia.edu.br)

Recebido em: 08/09/2015 - Aprovado em: 14/11/2015 - Publicado em: 01/12/2015 DOI: http://dx.doi.org/10.18677/Enciclopedia_Biosfera_2015_114

\begin{abstract}
RESUMO
As curvas de aprendizado são ferramentas favoráveis na monitoração do desempenho de um profissional submetido a uma nova tarefa, permitindo avaliar seu progresso na medida em que o mesmo realiza repetições. Neste estudo avaliou-se a curva de aprendizado em cirurgia de $\mathrm{OSH}$ em felinas realizada por acadêmicos do curso de Medicina Veterinária do Instituto Federal Catarinense- Câmpus Concórdia. O estudo envolveu 16 felinas de vida livre, com idade estimada entre 12 meses a quatro anos e com peso médio de $1,5 \pm 3,7 \mathrm{~kg}$. Os animais foram distribuídos em dois grupos de protocolo cirúrgico: grupo TTP (ligadura com fios cirúrgicos), e grupo TAB (ligadura com abraçadeira de náilon). No transoperatório de cada procedimento analisou-se o tempo de desempenho dos alunos-cirurgiões na execução de tarefas comparando as técnicas de $\mathrm{OSH}$ e a necessidade de intervenção docente. O maior tempo de aprendizado ocorreu durante o acesso ao ovário esquerdo utilizando a técnica de ligadura convencional $(T 4 p<0,05)$ em comparação com a técnica de abraçadeira que mostrou ser de fácil e rápida execução, diminuindo o tempo cirúrgico abdominal e redução do tempo de procedimento total $(T T p<0,05)$. A técnica de OSH em felinos usando abraçadeira de náilon mostrou-se viável e efetiva podendo ser usada em cirurgias rotineiras eletivas durante 0 aprendizado acadêmico.
\end{abstract}

PALAVRAS-CHAVE: ambiente acadêmico, curva de aprendizado, educação, felinas de vida livre, OSH.

\section{LEARNING CURVE COMPARISON BETWEEN CONVENTIONAL AND CLAMP OVARYSALPINGOHYSTERECTOMY TECHNIQUES IN ACADEMIC ENVIRONMENT}

\begin{abstract}
Learning curves are favorable tools in monitoring the performance of a professional subjected to a new task, allowing to evaluate his progress while he performs repetitions. This study evaluated the learning curve in feline ovariohisterectomy $(\mathrm{OSH})$ procedures conducted by students of the School of Veterinary Medicine from the Instituto Federal Catarinense, Câmpus Concordia. The study involved 16 free-
\end{abstract}


living animals, with estimated age from twelve months to four years and average weight of $1.5 \pm 3.7 \mathrm{~kg}$. The animals were divided into two groups, based on surgical protocols: TTP group (ligature with surgical sutures), and TAB group (ligature with nylon clamp). During each surgical procedure, the time performance in the execution of tasks by the students-surgeons was assessed, making possible the comparison between both surgical protocols and techniques, and the need for professor intervention in each case. There was a higher learning difficulty when accessing the left ovary and using the conventional ligature technique in comparison with the ligature with nylon clamp, which showed greater ease of execution and security, reducing the abdominal surgical time and allowing gradual reduction of the procedure total time $(\mathrm{TT} p<0,05)$. Therefore, the $\mathrm{OSH}$ technique in cats using nylon clamp proved to be feasible and effective to be used in routine elective surgeries.

KEYWORDS: academic environment, learning curve, education, free-living felines, $\mathrm{OSH}$.

\section{INTRODUÇÃO}

Conceitos teóricos e práticos envolvem o ambiente do ensino cirúrgico, as fases fundamentadas e o desenvolvimento de habilidades são considerados indispensáveis no aprendizado acadêmico (MOREIRA \& MARTINS, 2012). Para tal, são desenvolvidas repetições e observações onde o estudante além de acompanhar a rotina cirúrgica, realiza procedimentos operatórios experimentais em animais vivos, cadáveres ou em bonecos modelos para adquirir competência, por tentativas e erros (SMEAK, 2003).

Os conhecimentos obtidos através da prática da cirurgia são acompanhados por mestres, entretanto, a ampla fundamentação teórica exigida na formação, assim como o número restrito de professores nas universidades são fatores limitantes ao treinamento dos alunos prejudicando o desenvolvimento das habilidades essenciais para uma boa evolução acadêmica (JENKINS et al., 2008). Essa atual diversidade requer um novo direcionamento no ensino através de meios didáticos inovadores e experimentais (BUYUKMIHCI, 2007). Por imediato, para garantir a aquisição de habilidades, diversos métodos alternativos e que projetem rapidez têm sido desenvolvidos, buscando-se cumprir a função educacional (CAMPOS \& ROCHA, 1996).

As maiores dificuldades acadêmicas são expressas na fase inicial de aprendizado que é constituído pela ânsia em esclarecer dúvidas perante uma série de situações que são demonstradas e vivenciadas. Dentre os procedimentos cirúrgicos de iniciação, a OSH é um dos primeiros procedimentos praticados intensamente na disciplina cirúrgica e posteriormente realizados com maior frequência na rotina operatória (SOARES \& SILVA, 1998; BASSO et al., 2014).

Essa técnica cirúrgica realizada em fêmeas representa uma modalidade didática nova para o estudante principiante. Como esses alunos não dominam a metodologia, ao praticá-la ocorre individualmente dificuldades que prolongam o tempo operatório no animal (BARROS et al., 2009). Segundo PINTO FILHO et al. (2014), essas dificuldades estão relacionadas à longa curva de aprendizado, a complexidade da técnica invasiva e a falta de habilidade.

As curvas de aprendizado têm sido utilizadas como ferramenta para avaliação do tempo demandado para conclusão de uma tarefa. A formulação de uma curva leva em consideração alguns fatores que podem influenciar o progresso, tais como: método de treinamento do procedimento, motivação individual, conhecimento prévio e número de casos necessários para realizar um procedimento 
em tempo operatório adequado (NEMBHARD \& OSOTHSILP, 2002; SEREL et al., 2003).

A medição do desempenho de um estudante na execução de uma tarefa pode ser feita através da observação das variáveis: tempo, demanda, número de repetições e a proficiência que é o momento em que o aluno-cirurgião pode realizar o procedimento sem supervisão (SEREL et al., 2003; BASSO et al., 2014). Outro fator importante no treinamento de alunos está em realizá-lo em animais vivos e durante as aulas práticas, proporcionando ao futuro cirurgião a percepção real da situação cirúrgica que deverá enfrentar (BARROS et al., 2009). De acordo com PINTO FILHO et al., (2014) e RASHAL \& INÁCIO (1995), a maioria dos acadêmicos não revelam problemas em participar de aulas práticas que utilizam animais vivos e acreditam que métodos alternativos são eficientes para um aprendizado de qualidade.

Há poucos relatos na literatura atual acerca do estudo de curva de aprendizado em procedimentos cirúrgicos realizados em felinos. Considerando-se a importância da informação sobre as habilidades do aluno-cirurgião em relação ao tempo operatório, este trabalho objetivou comparar a curva de aprendizagem durante a realização da cirurgia principiante de OSH convencional e com a metodologia utilizando a abraçadeira de náilon em felinas, em ambiente acadêmico de aprendizagem e observar a necessidade da intervenção docente durante o transoperatório.

\section{MATERIAL E MÉTODOS}

\section{Animais}

Foram utilizadas 16 felinos de vida livre, sem raça definida (SRD), com peso médio de $1,5 \pm 3,7 \mathrm{~kg}$, idade estimada entre 12 meses a quatro anos, comprovadamente hígidas que transitavam livremente no Campus do IFCConcórdia. Os animais foram distribuídos aleatoriamente em dois grupos de protocolo cirúrgico, com mesmo número de animais: grupo TTP (ligadura com fios cirúrgicos) e grupo TAB (ligadura com abraçadeira de náilon 6.6, com dimensões de $2,5 \times 80 \mathrm{~mm}$.). Os animais foram vermifugados e mantidos em gatis individuais enriquecidos ambientalmente por 15 dias para padronização dos cuidados da alimentação e socialização humana.

\section{Comitê em ética}

O estudo foi submetido à aprovação do Comitê de Ética e Experimentação Animal do Instituto Federal Catarinense - Campus Concórdia, seguindo os princípios éticos do Colégio Brasileiro de Experimentação Animal (COBEA). Protocolo CEUA número 28/2014.

\section{Local de estudo}

Os procedimentos cirúrgicos foram realizados no Centro Prático Clínico e Cirúrgico do IFC-Concórdia, na disciplina de Técnica Cirúrgica. Os pacientes foram submetidos a exame clínico geral e laboratorial, pré-operatório, sendo excluídos do experimento os animais comprometidos.

\section{Procedimento anestésico}

Os animais foram submetidos a jejum alimentar de 12 horas e hídrico de 6 horas antecedendo o dia do experimento. Receberam como medicação pré- 
anestésica (MPA) cetamina $S(+)\left(5 \mathrm{mg} \cdot \mathrm{kg}^{-1}\right)$ associada ao midazolam $\left(0,5 \mathrm{mg} \cdot \mathrm{kg}^{-1}\right)$, pela via intramuscular (IM). Após 15 minutos, foi administrado propofol $\left(4 \mathrm{mg} \cdot \mathrm{kg}^{-1}\right)$, pela via intravenosa (IV) e intubação orotraqueal para fornecimento oxigênio a 99\% e anestesiados com halotano durante o período de manutenção anestésica e cirúrgica. Para anestesia epidural foi administrada morfina $\left(0,1 \mathrm{mg} \cdot \mathrm{kg}^{-1}\right)$ e bupivacaína $\left(0,25 \mathrm{mg} \cdot \mathrm{kg}^{-1}\right)$ associadas. Como medicação pré emptiva os animais receberam, ampicilina $\left(20 \mathrm{mg} \cdot \mathrm{kg}^{-1}\right)$; meloxican $\left(0,2 \mathrm{mg} \cdot \mathrm{kg}^{-1}\right)$; tramadol $\left(2 \mathrm{mg} \cdot \mathrm{kg}^{-1}\right)$.

\section{Procedimento cirúrgico Grupo TTP}

Os pacientes foram posicionados em decúbito dorsal e a região ventral tricotomizada para cirurgia asséptica e então submetidas a celiotomia por meio de incisão mediana ventral, localizada um centímetro caudal a cicatriz umbilical, estendendo-se cerca de cinco centímetros caudalmente ao abdome. Após incisão de pele e linha alba, com auxílio de gancho de Cavault, foi exposto o corno uterino direito, gentilmente, para elevá-lo até a cavidade abdominal. Após observação anatômica, foi identificado o ligamento suspensório e logo em seguida ele foi rompido para permitir a exteriorização do ovário direito. Efetuou-se um orifício no ligamento largo caudal ao pedículo ovariano para o posicionamento de duas pinças de Rochester-Carmalt, no pedículo ovariano proximal até o ovário e uma no ligamento próprio do ovário. O pedículo ovariano entre a pinça média e o ovário foi transeccionado e a sutura em "oito" com fio poliglatina 910 (2.0), proximal às pinças do pedículo foi realizada como ligadura. A pinça proximal foi removida enquanto a ligadura foi apertada para permitir a compressão do pedículo. Após, foi realizada a ressecção do corno uterino, seguida de observação sobre a possibilidade de sangramento durante 60 segundos marcados. Foi realizado o mesmo procedimento no ovário oposto. Posteriormente, foi tracionado o corpo do útero que foi ligado com sutura 'em oito' cranialmente à cervix. Uma ligadura circular foi realizada mais próxima a cérvix. Após as ligaduras o coto observou-se por 60 segundos para constatação da possibilidade de sangramento, seguida da omentopexia do coto com fio poliglatina 910 (2.0). A laparorrafia foi realizada respeitando os planos anatômicos empregando-se sutura de sultan com fio mononailon (2.0), aproximação do espaço morto com fio poliglatina 910 (2.0) em sutura contínua e sutura de pele com mononailon (2.0) em sutura intradérmica.

\section{Procedimento cirúrgico Grupo TAB}

Os pacientes foram posicionados em decúbito dorsal e a região ventral tricotomizada para cirurgia asséptica e então submetidas a celiotomia por meio de incisão mediana ventral, localizada um centímetro caudal a cicatriz umbilical, estendendo-se cerca de cinco centímetros caudalmente ao abdome. O corpo do útero foi identificado com auxílio de gancho de Cavault, guiado-se pela sua localização dorsal à bexiga e ventral ao cólon. Seguindo-se à direção cranial, os cornos direito e esquerdo foram identificados. O corno uterino direito foi gentilmente exteriorizado mediante tração ventral do órgão e compressão dorsal da parede abdominal direita com afastador de Farabeuf. Com o ligamento suspensor distendido, a bursa ovárica e o complexo artéria-veia ovariana (CAVO) identificados, foi realizada a ruptura do ligamento largo na região do mesovário. Uma única abraçadeira foi colocada em torno do complexo CAVO e do ligamento suspensor, o sistema de travagem foi acionado, proporcionando progressiva compressão circular e fixação da mesma em um ponto cranial da bursa ovárica. A secção foi realizada entre a ligadura e a presença ou não de sangramento, avaliada em um período 
marcado de 60 segundos. Constatada a hemostasia, foi realizada a secção transversal da fita, imediatamente após a saída do sistema de travagem. O corno uterino foi retrofletido e o ligamento largo correspondente rompido. A partir do deslocamento, foi identificado o corno colateral e as manobras operatórias foram novamente executadas para obliteração do pedículo ovariano esquerdo.

Com o corpo do útero exposto, foi colocada uma única abraçadeira em torno do mesmo, o sistema de travagem foi acionado, proporcionando progressiva compressão circular e fixação da mesma na região cranial a cérvix. O corpo do útero foi seccionado entre as ligaduras e a presença ou não de sangramento, avaliada por um período marcado de 60 segundos. Efetuada a hemostasia, realizou-se a secção transversal da fita, longitudinalmente ao eixo das hastes imediatamente após a saída do sistema de travagem (BARROS et al., 2009). Adicionalmente foi realizada a omentopexia sobre o coto uterino empregando-se um ponto de reparo com fio poliglatina 910 (2.0). A laparorrafia foi realizada respeitando os planos anatômicos empregando-se fio de sutura de sultan com mononailon, aproximação do espaço morto com fio poliglatina 910 (2.0) em sutura contínua e sutura de pele com mononailon (2.0) com sutura intradérmica.

No pós-operatório foi realizado terapia antiinflamatória com meloxican $(0,2 \mathrm{mg} \cdot \mathrm{kg}$ $\left.{ }^{1}\right)$, via SC, BID; tramadol $\left(2.0 \mathrm{mg} \mathrm{kg}^{-1}\right)$ SID, por três dias, via SC. As incisões foram higienizadas diariamente com solução fisiológica ( $\mathrm{NaCl}$ 0,9\%). Após 10 dias de observação clínica e retirada dos pontos, os animais foram doados.

\section{Delineamento experimental}

Antes da realização do primeiro e do segundo grupo cirúrgico foi realizado treinamento teórico-prático demonstrativo previsto na disciplina de técnica cirúrgica, reforçando o conteúdo nas aulas teóricas. Os animais experimentais foram separados em dois grupos, com oito componentes cada, distribuídas aleatoriamente e sequencialmente. Todas as cirurgias foram realizadas por acadêmicos cirurgiões em esquema de rodízio entre os mesmos membros de equipes para que pudesse ser avaliada a curva de aprendizado em relação ao tempo dispendido da equipe cirúrgica composto por quatro alunos como um grupo de organização com ou sem necessidade de intervenção do professor.

Em cada procedimento foi analisado o tempo de desempenho na execução de tarefas comparando $\mathrm{OSH}$ pela técnica convencional de ligadura e pela técnica de abraçadeira de náilon. Os tempos de execução avaliados foram: tempo zero (T0), momento da incisão de pele até acesso à cavidade abdominal; tempo 1 (T1), reconhecimento das estruturas anatômicas abdominais e tração do ovário direito; tempo 2 (T2), momento da ligadura e remoção do ovário direito; tempo 3 (T3), reconhecimento das estruturas anatômicas abdominais e tração do ovário esquerdo; tempo 4 (T4), ligadura e remoção do ovário esquerdo; tempo 5 (T5), reconhecimento das estruturas anatômicas abdominais e localização do corpo do útero/cérvix; tempo 6 (T6), ligadura e remoção do corpo uterino/cérvix; tempo 7 (T7), sutura dos planos da cavidade abdominal; tempo 8 (T8), sutura da pele; tempo final (TF); e tempo total (TT).

Os resultados coletados foram transferidos para uma planilha em excel, e as análises estatísticas foram realizadas por análises descritivas e a análise de variância (ANOVA). 


\section{RESULTADOS E DISCUSSÃO}

A escolha pela espécie felina baseou-se na necessidade de informações sobre a espécie que vive em vida livre, pela praticidade e rapidez que o procedimento oferece nessa espécie (SCHIOCHET et al., 2009) e pela necessidade de castrar as fêmeas que perambulam no câmpus da Instituição.

A cirurgia de OSH não configura uma nova especialidade operatória (BASSO et al., 2014), mas para o acadêmico cirurgião iniciante é a cirurgia que necessita de uma fase de adaptação, e, portanto, necessita de treinamentos específicos associado a habilidade manual, coordenação psicomotora e visual (SOARES \& SILVA, 1998). No presente estudo, a curva de aprendizado mostrou-se satisfatória, apresentando maiores dificuldades quando da necessidade de fechamento dos planos da parede abdominal.

Para realizar o estudo foi necessário, em ambas as técnicas, acessar a cavidade abdominal para promover uma adequada visualização da anatomia abdominal. No experimento optou-se pela técnica aberta de exposição visceral por ser considerada de fácil aplicabilidade na rotina médica veterinária e pelo fato dos felinos apresentarem uma parece abdominal extremamente delgada assim como a cavidade abdominal pequena favorecendo a manipulação aos dois grupos quanto ao tempo de aprendizado. A manobra realizada nos grupos TTP e TAB mostrou-se bastante efetiva. A TAB permitiu adequada visualização dos vasos ovarianos e espaço suficiente para a realização da ligadura por pressão, evitando a colocação de uma segunda ou terceira ligadura.

O tempo total de duração do procedimento no grupo de OSH por técnica de ligadura de fios (TTP) variou de 99 a 150 minutos, com tempo médio variando de $116,25 \pm 18,22$ (Tabela 1). O tempo cirúrgico do grupo que realizou OSH por técnica de abraçadeira de náilon (TAB) variou de 53 a 100 minutos, tempo médio de cirurgia de $77,5 \pm 17,3(p<0,05)$ (Tabela 1) Percebeu-se nesse trabalho que cirurgia de $\mathrm{OSH}$ convencional (TTP), para o acadêmico em aprendizado foi o procedimento que demandou maior atenção e tempo dispendido por não terem o domínio da prática, ou ainda, da habilidade individual do cirurgião, mas que exigiu igualmente em ambas as técnicas uma concentração e nova maneira de aprender. Comparando-se a equipe cirúrgica em relação às duas técnicas apresentadas observou-se que o tempo total (TT) de duração do procedimento foi maior no grupo TTP $(P<0,05)$ do que no TAB. A utilização dos instrumentos cirúrgicos e fios que demandam técnica anatômica (TTP), ainda não adaptada pelo cirurgião, a tensão e medo contribuíram para esse prolongamento do tempo operatório, observados também por SCHIOCHET et al., (2009), além de que o tempo dispendido para colocação do artefato abraçadeira de náilon demandou menor tempo em T2 e T4, diminuindo o tempo final do procedimento no grupo TAB.

A representação da curva de aprendizado (Tabela 1 ) demonstra a média comparativa entre todos os tempos cirúrgicos e o TT das técnicas de ligadura por fios e abraçadeira de náilon. Observou-se que nos tempos T1 (incisão de pele até o acesso à cavidade abdominal); T2 (localização e tração do ovário direito) e T3 (ligadura e remoção do ovário direito) não ocorreram diferença significativa entre os grupos, entretanto, no momento T4 percebeu-se diferença $(p<0,05)$ sendo que 0 grupo TAB demonstrou maior rapidez ao realizar a ligadura e remoção no ovário esquerdo. O grupo TTP apresentou maior demora na realização do procedimento direcionado e na maioria dos tempos $(70 \%)$ ocorreu necessidade de intervenção docente para finalização do processo, muitas vezes devido a insegurança na localização anatômica e no modo de colocação dos fios para hemostasia. Percebeu- 
se também que devido o aluno ter que estender os braços para o alcance do ovário esquerdo, a presença de gordura na bolsa ovariana e a dificuldade na movimentação manual dos instrumentos cirúrgicos prolongaram o período transoperatório. Esse tempo poderia ser menor se ocorresse melhor comunicação entre o cirurgião e o auxiliar, fato esse pouco observado entre os tempos. No grupo TAB a intervenção docente ocorreu em $40 \%$ dos tempos e na maioria dos chamados foi devido à incerteza sobre força de pressão para o travamento das abraçadeiras para a devida hemostasia dos cotos.

Este é um período crítico para o cirurgião, dos oito felinos de cada grupo, quatro deles (3-TTP e 1-TAB) o cirurgião perdeu o coto, todavia, com a intervenção e auxílio do docente, o contratempo foi reparado imediatamente. Estas lesões acontecem devido a ligadura não ter sido completa ou ainda na falta de agilidade para o reposicionamento de pinças hemostáticas (SOUZA et al., 2014) ou da abraçadeira. Outro fator de risco é o sangramento intra-operatório de difícil controle, variações anatômicas ou idade avançada (SANTOS, 2010; FERANTI et al., 2015), nesse estudo ocorreram sangramento nos quatro casos citados, porém, não foi evidenciado transtornos anatômicos ou alterações relacionadas com a idade dos pacientes.

Considerando os fatores de risco que estão presentes no período de treinamento, nem sempre ocorre entre os universitários a fase de treinamento nas práticas de cada nova cirurgia. O cirurgião nem sempre é capaz de reconhecer as variações anatômicas e isso faz com que ocorram lesões por insegurança ou ainda processo inflamatório intenso (BASSO et al., 2014). Essas condições foram avaliadas na pesquisa onde se percebe que acadêmicos que passaram por treinamento adquirem maior segurança pessoal para a realização de um próximo procedimento.

Observou-se estatisticamente que no momento entre T8 até a finalização (Tf) referente ao tempo de realização da sutura dos planos abdominais, o acadêmico em treinamento já possuía uma habilidade e agilidade instrumental nos grupos TAB e TTP comparando com tempo T1, entretanto, pelo fato de o grupo TTP ter manuseado por mais tempo os instrumentais cirúrgicos, quando em Tf já tinha agilidade e rapidez, apresentando diferença significativa $(p<0,05)$ (Tabela 1) no tempo operatório comparados ao grupo TAB. SILVA et al. (2006) perceberam que o tempo operatório também foi reduzido quando usaram abraçadeiras de náilon em procedimentos de orquiectomia em equinos e não observaram alterações fisiopatológicas nos animais durante o pós operatório.

Embora seja considerada uma desvantagem a duração maior (TT) do procedimento realizado em felinos em TTP, já era esperada esses dados pois, levase algum tempo para conhecer adequadamente a anatomia regional dos cotos $\mathrm{e}$ aplicar a técnica de ligadura e ainda a desvantagem quanto ao tempo pode aumentar com a falta de habilidade e experiência do cirurgião, bem como o nervosismo e tensão do momento (BARROS et al. , 2009; SANTOS, 2010).

A ausência de complicações pós-operatória imediata e tardia foi observada em todos os grupos dos animais. Para uma maior segurança deve-se empregar rigorosamente uma força para fixação da abraçadeira seguida de corte das hastes proximal ao anel da abraçadeira, impedindo qualquer segmento traumático aos tecidos adjacentes ao coto ovariano e uterino (BARROS et al., 2009). Se a força empregada para a fixação do artefato não atender a necessidade de hemostasia será facilmente observado sangramento. Nos casos avaliados, ocorreu uma soltura do pedículo ovariano, sendo este no ovário esquerdo de um dos pacientes do grupo 
TAB. Para atender a correção e reverter o sangramento optou-se por introduzir uma nova abraçadeira, abaixo da primeira assegurando assim a segurança e obliteração vascular. E importante enfatizar a necessidade de se manter a secção do pedículo ovariano uma margem de segurança e a observação por no mínimo três minutos com os cotos relaxados, atendendo a prioridade de verificação da nulidade de sangramento local (OLIVEIRA, 2006).

TABELA 1: Número de animais (n), valores mínimos e máximos, média, erro padrão, desvio padrão, coeficiente de variação (CV \%), valor de p da análise estatística descritiva e análise de variância $\left(p \leq 0,05^{*}\right)$ entre os tempos e entre os grupos (ANOVA).

\begin{tabular}{|c|c|c|c|c|c|c|c|c|c|}
\hline Variável & $\mathrm{n}$ & Tratamento & Mínimo & Máximo & Média & $\begin{array}{c}\text { Erro } \\
\text { Padrão }\end{array}$ & $\begin{array}{l}\text { Desvio } \\
\text { Padrão }\end{array}$ & $\begin{array}{l}\text { CV } \\
(\%)\end{array}$ & Valor-p \\
\hline \multirow[t]{2}{*}{$\mathrm{T} 1$} & 8 & 1 & 3.00 & 13.00 & 5.88 & 1.17 & 3.31 & 56.41 & \\
\hline & 8 & 2 & 2.000 & 7.000 & 3.625 & 0.565 & 1.598 & 44.08 & \\
\hline \multirow[t]{2}{*}{$\mathrm{T} 2$} & 8 & 1 & 6.00 & 28.00 & 14.50 & 2.67 & 7.54 & 52.00 & 0.106 \\
\hline & 8 & 2 & 2.00 & 23.00 & 9.25 & 2.43 & 6.86 & 74.17 & \\
\hline \multirow[t]{2}{*}{ T3 } & 8 & 1 & 1.00 & 16.00 & 10.50 & 1.70 & 4.81 & 45.82 & 0.167 \\
\hline & 8 & 2 & 2.000 & 10.000 & 5.125 & 0.972 & 2.748 & 53.63 & \\
\hline \multirow[t]{2}{*}{$\mathrm{T} 4$} & 8 & 1 & 1.00 & 16.00 & 6.50 & 2.15 & 6.07 & 93.40 & $0.016^{*}$ \\
\hline & 8 & 2 & 1.00 & 9.00 & 3.25 & 1.05 & 2.96 & 91.20 & \\
\hline \multirow[t]{2}{*}{ T5 } & 8 & 1 & 5.000 & 11.000 & 8.250 & 0.750 & 2.121 & 25.71 & 0.195 \\
\hline & 8 & 2 & 2.00 & 11.00 & 4.38 & 1.03 & 2.92 & 66.85 & \\
\hline \multirow[t]{2}{*}{ T6 } & 8 & 1 & 1.00 & 9.00 & 3.38 & 1.13 & 3.20 & 94.94 & 0.194 \\
\hline & 8 & 2 & 1.000 & 4.000 & 1.750 & 0.366 & 1.035 & 59.15 & \\
\hline \multirow[t]{2}{*}{ T7 } & 8 & 1 & 6.00 & 32.00 & 16.13 & 3.36 & 9.51 & 58.97 & \\
\hline & 8 & 2 & 1.00 & 13.00 & 5.88 & 1.39 & 3.94 & 67.13 & $0.014^{\wedge}$ \\
\hline \multirow[t]{2}{*}{ T8 } & 8 & 1 & 15.00 & 40.00 & 26.50 & 3.20 & 9.04 & 34.11 & 0.266 \\
\hline & 8 & 2 & 19.00 & 51.00 & 32.50 & 4.07 & 11.51 & 35.43 & \\
\hline \multirow[t]{2}{*}{$\mathrm{Tf}$} & 8 & 1 & 7.00 & 27.00 & 17.13 & 2.14 & 6.06 & 35.37 & $0.051^{*}$ \\
\hline & 8 & 2 & 8.00 & 20.00 & 11.75 & 1.33 & 3.77 & 32.09 & \\
\hline \multirow[t]{2}{*}{ TT } & 8 & 1 & 99.00 & 150.00 & $\begin{array}{c}116.2 \\
5\end{array}$ & 6.44 & 18.22 & 15.67 & 0.001 * \\
\hline & 8 & 2 & 53.00 & 100.00 & 77.50 & 6.15 & 17.39 & 22.43 & \\
\hline
\end{tabular}

Quanto à utilização das abraçadeiras de náilon em cirurgia, alguns trabalhos científicos produzidos nos últimos anos propuseram a utilização de artefatos de poliamida em vídeocirurgias (CALDAS et al., 2015) ou cirurgias convencionais, nos quais testaram o comportamento biológico do implante, e em cães submetidos a cirurgias para ligaduras vasculares e intestinais. Os estudos constataram pequena reação tecidual à presença do implante e confirmaram a exequibilidade, praticidade, segurança e economia na utilização das mesmas, sugerindo que possam ser aceitas como método alternativo na prática cirúrgica (BARROS et al., 2009). Os pacientes dos grupos TTP e TAB deste estudo foram avaliados clinicamente quanto ao 
comportamento fisiológico por um período de 10 dias não sendo observadas alterações na micção, no apetite, na dor local, na defecação e nas atitudes lúdicas.

Segundo RAHAL et al., (1999) ocorre reação tecidual induzida por sutura com fio de náilon quando realizada em cotos ovarianos e uterinos mas que evolui gradativamente para uma cápsula delgada de tecido conjuntivo fibroso após procedimento cirúrgico. Baseando-se nesses resultados existe uma similaridade na composição química entre fios de náilon e fitas de poliamida supondo o mesmo comportamento orgânico entre eles. No grupo TTP as ligaduras dos cotos ovariano e uterino foram realizadas com fios absorvíveis não sendo observada nenhuma alteração pós-operatória.

A qualidade do desempenho do cirurgião muda no decorrer do procedimento. Iniciam temerosos, mas, no final da cirurgia já adquirem segurança pessoal (SANTOS , 2010). A participação do docente ou a sua proximidade na sala de aula passa segurança ao acadêmico em treinamento. Esse treinamento foi considerado adequado e capaz de melhorar o desempenho do aluno com ganho de desenvoltura e a experiência aplicada nos animais. Também foi fundamental para a obtenção de bons resultados, porém, não eliminou os fatores de riscos e lesões promovidas pelo próprio cirurgião. De acordo com MATERA (2008) o uso de animais em aulas práticas também traz à tona aspectos importantes para a formação do caráter do indivíduo. O acadêmico vê na figura do educador o exemplo, não se tratando apenas de uma relação superficial, mas, seguindo suas orientações. Neste estudo percebeu-se que o docente ao se afastar da visão do aluno-cirurgião este em questão de minutos solicitava sua presença em seu campo visual.

\section{CONCLUSÕES}

A curva de aprendizado é um procedimento didático eficiente para ser adotado na prática cirúrgica. A técnica de $\mathrm{OSH}$ em felinos usando abraçadeira de náilon mostrou-se viável e efetiva podendo ser utilizada em cirurgias rotineiras eletivas e campanhas de castração diminuindo o tempo cirúrgico. A maioria dos acadêmicos principiantes prefere que o docente permaneça próximo a mesa cirúrgica durante o transoperatório.

\section{REFERÊNCIAS}

BARROS, B. J; SANCHES, A. W. D; PACHALY, J. R. Utilização de abraçadeiras de náilon 6.6 (poliamida) como método de ligadura de pedículos ovarianos e coto uterino em ovário-histerectomia eletiva em cadelas (canis familiaris). Arquivos de Ciências Veterinárias e Zoologia da Unipar, Umuarama, v. 12, n. 1, p. 47-60, jan./jun. 2009.

BASSO, P. C, RAISER, A. G., BRUN, M. V., MAZZANTI, C. M., COSTA, M. M., WOLKMER, P., DUTRA, L.H., MÜLLER, D. C. M.,SILVA, C. B. Biomarcadores inflamatórios e indicadores de estresse oxidativo em cadelas submetidas à ovariossalpingohisterectomia convencional, por NOTES híbrida e NOTES total. Ciência Rural, v.44, n.5, p.884-890, 2014.

BUYUKMIHCI, N.C. Non-violence in surgical training. Revista de Eletrônica de Veterinária, v.8, n.12, 2007. 
CALDAS, P. F.O., ROCHA, L. C. A., OLIVEIRA, M. T. A., WESTPHAL M., SPAGNOLO, J. D., FERANTI, J. P. S., SOUZA, F. W., SILVA, M. A. M., BRUN, M. V. Laparoscopic ureterocalicostomy in pigs - experimental study. Ciência Rural, v.45, n.7, p.1262-1267. Epub 28 de abril de 2015.

CAMPOS, G. H. B., ROCHA, A. R. C. Dez etapas para o desenvolvimento de software educacional do tipo hipermidia. In: $3^{\circ}$ CONGRESSO IBERAMERICANO DE INFORMATICA EDUCATIVA, 1996, Barranquilla. Anais... Barranquilla: [v.1] 1996. (Resumo Expandido).

FERANTI, J. P.S., OLIVEIRA, M. T. SOUZA, F. W., CORRÊA, L. F. DUTRA, P. F., SAULO T. L., DALMOLIN, F., HARTMANN, H. F.,BRUN, M. V. Laparoendoscopic Single-Site Surgery na realização de criptorquidectomia laparoscópica em um gato. Ciência Rural, , 00. Epub 10 de julho de 2015.

FILHO, S.A.V.P., ARRUDA, E.F., GOMES, F.A., SOUZA, S.F., FEIRAS, H.J. Uso de animais em aulas práticas: perfil e percepção dos Acadêmicos do curso de medicina veterinária da UFAC. Enciclopédia biosfera , Centro Científico Conhecer - Goiânia, v.10, n.19; p.1821, 2014.

JENKINS, S., GOEL, R., MORRELL,D.S. et al. Computer-assisted instruction versus traditional lecture for medical student teaching of dermatology morphology: Arandomized control trial. Journal of the American Academy of Dermatology, v.59, n.2, 2008.

MATERA, J. M. Método de Ensino Substitutivo na Disciplina de Técnica Cirúrgica. In: Instrumento animal: o uso prejudicial de animais no ensino superior. Bauru, SP: Canal 6, 2008. p. 203-204

MOREIRA, J.C.N., MARTINS, E.F.F. Uso de animais para o ensino da cirurgia na medicina veterinária. Qual a alternativa? Conselho Federal de Medicina Veterinária. Disponível em: $<$ http://www.cfmv.gov.br/portal/inscricao df/material/dia 15/USO\%20DE\%20ANIMAI S\%20PARA\%200\%20ENSINO\%20DA\%20CIRURGIA\%20NA\%20MEDICINA\%20V ETERINARIA.\%20\%20QUAL\%20A\%20ALTERNATIVA.pdf>. Acesso em 12 de março de 2015.

NEMBHARD, D. A.; OSOTHSILP, N. Task Complexity Affects on Between-Individual Learning/Forgetting Variability. International Journal of Industrial Ergonomics, London, v. 29, n.5, p. 297-306, 2002.

OLIVEIRA, M. S. Utilização de abraçadeiras de náilon para hemostasia preventiva na ováriosalpingohisterectomia em gatas. 2006. 43 f. Trabalho de conclusão de curso (Graduação em Medicina Veterinária) - Universidade Federal da Bahia, Salvador, 2006.

PINTO FILHO, S. T. L., DALMOLIN, F., OLIVEIRA, M.T, SOUZA, F. W., SPRADA, A. G., ROSA, M. P., FERANTI, J. P. S., BRUN, M. V. Associated laparoscopic-assisted 
gastropexy and ovariohisterectomy in a Great Dane bitch. Ciência Rural, v. 45, n.2, p. 280-283. 2014.

RAHAL, S.C., GUIMARÃES, M.LANGONI, H., FORTES, J.V., LANGONI, H. , LUCCHIARI, P.H. Estudo comparativo entre o fio de náilon e a linha de pesca(poliamida) e o fio de náilon ciúrgico e a sua aplicabilidade como fio cirúrgico. Archival of Veterinary Science, Curitiba. V, 4, n.1, 1999, p.89-94.

RASHAL, F.; INÁCIO, V. Treinamento em videocirurgia. In: COELHO, J.C.U. et al. (Eds.). Complicações da videocirurgia: da profilaxia ao tratamento. Rio de Janeiro: MEDSI, 1995. p.11-16.

SANTOS, E.G. Curva de aprendizado e lesões iatrogênicas em colecistectomias videolaparoscópicas. Revista do Colégio Brasileiro de Cirurgia. v.37, n.3, p.184189, 2010.

SCHIOCHET, F., BECK, C.A.C., SILVA, A.P.F.F., CONTESINI, E.A., ALIEVI, M.M., STEDILE, R.,PINTO, V., YAMASAKI, P.H., JURINITZ, D.F., PELLIZARI, M. Ovariohisterectomia laparoscópica em felinos hígidos: estudo comparativo de três métodos de hemostasia. Arquivo Brasileiro de Medicina Veterinária e Zootecnia, v.61, n.2, p.369-377, 2009.

SEREL, D. A., DADA, M., MOSKOWITZ, H. PLANTE, R.. Investing in Quality Under Autonomous and Induced Learning. IIE Transactions, London, v. 32, p. 545-555, 2003.

SILVA, L. A. F.; FRANÇA, R. O.; VIEIRA, D.; SOUZA, V. R.; FRANCO, L. G.; MOURA, M. I.; SILVA, M. A. M.; TRINDADE, B. R.; COSTA, G. L.; BERNARDES, K. M. Emprego da abraçadeira de náilon na orquiectomia em eqüinos. Acta Scientiae Veterinarie, n.34, p.261-266, 2006.

SMEAK D. D. Ethical surgical training for students of veterinary medicine In.JUKES, N.; CHIUIA, M. From Guinea Pig to Computer Mouse: Alternative Methods for Progressive, Humane Education, 2nd Ed. Leicester, UK: InterNICHE, 2003.

SOARES, J.A.G.; SILVA, P.A.R. Castração precoce em cães e gatos. Clínica Veterinaria, Ano III, n.13, p.34-40, 1998.

SOUZA, F. W., BRUN, M. V., OLIVEIRA, M. T., FERANTI, J. P. S., CORRÊA, R. KA. R., IDALÊNCIO, R., D., NAILA C. B., QUADROS, A. M.,HUPPES, R. R. Ovariohisterectomia por videocirurgia (via NOTES vaginal híbrida), celiotomia ou miniceliotomia em cadelas. Ciência Rural, v.44, n.3, p. 510-516, 2014. 
